\title{
PÍLULA DO DIA SEGUINTE: IMPORTÂNCIA DA ATENÇÃO FARMACÊUTICA NO USO DE CONTRACETIVO DE EMERGÊNCIA PARA AS ADOLESCENTES
}

\author{
Walace Rodrigues Costa ${ }^{1}$ \\ Fabiana Sousa Pugliese ${ }^{2}$ \\ Michel Santos da Silva ${ }^{3}$ \\ Leonardo Guimarães de Andrade ${ }^{4}$
}

RESUMO: A pílula do dia seguinte (PDS) é chamada pelos médicos de pílula anticoncepcional pós-coito. É um método anticoncepcional de emergência e deve ser tomada até 72 horas após a relação sexual. É adequado para casos de abuso sexual, preservativos quebrados ou falha de outros métodos anticoncepcionais. É composto dos mesmos hormônios que os anticoncepcionais, mas em uma dose um pouco maior, você pode encontrar anticoncepcionais em uma caixa, duas caixas ou quatro caixas. Esta pílula só pode ser vendida com receita médica porque contém altas doses de hormônios, o que pode causar graves consequências para o corpo das mulheres. É muito importante que as mulheres usem anticoncepcionais ambulatoriais apenas em emergências e não como método anticoncepcional. Mulheres que fazem sexo com frequência podem usar outros métodos anticoncepcionais, como preservativos, dispositivos intrauterinos e anticoncepcionais. Eles são prescritos por médicos em quem eles confiam. Lembre-se de que a Organização Mundial da Saúde recomenda o uso de anticoncepcionais para evitar a gravidez e preservativos para prevenir DST (Doença Sexualmente Transmissível). Seu efeito não é tão bom quanto o dos anticoncepcionais comumente usados na vida diária e varia com o tempo de uso. A equação é a seguinte: quanto maior o atraso na ingestão, maior a chance de gravidez.

Palavras- chaves pílula do dia seguinte. Anticoncepcional. Hormônios.

ABSTRACT: The morning-after pill (PDS) is called the postcoital contraceptive pill by doctors. It is an emergency contraceptive method and must be taken within 72 hours of intercourse. It is suitable for cases of sexual abuse, broken condoms, or failure of other contraceptive methods. It's made up of the same hormones as contraceptives, but at a slightly higher dose, you can find contraceptives in one box, two boxes, or four boxes. This pill can only be sold with a prescription because it contains high doses of hormones, which can have serious consequences for women's bodies. It is very

\footnotetext{
I Graduação em Farmácia pela Universidade Iguaçu. E-mail: walacerodriguescosta@gmail.com.

${ }^{2}$ Farmacêutica.

3 Biólogo.

4 Enfermeiro/odontologia pela Universidade Iguaçu.
} 
important that women use outpatient contraceptives only in emergencies and not as a contraceptive method. Women who have sex frequently can use other contraceptive methods such as condoms, intrauterine devices and contraceptives. They are prescribed by doctors they trust. Remember that the World Health Organization recommends the use of contraceptives to prevent pregnancy and condoms to prevent STDs (Sexually Transmitted Disease). Its effect is not as good as that of contraceptives commonly used in daily life and varies with time of use. The equation is as follows: the longer the delay in ingestion, the greater the chance of pregnancy.

Keywords: Morning-after pill. Contraceptive. Hormones.

\section{INTRODUÇÃO}

A pílula ambulatorial é um método anticoncepcional de emergência, na forma de pílula, que pode ser usada após a relação sexual, quando uma mulher imagina ter relações sexuais sem tomar os devidos cuidados, pode inibir a gravidez. Embora o Ministério da Saúde tenha facilitado sua distribuição, ainda existem dificuldades na obtenção de informações, principalmente informações sobre o uso correto dos medicamentos e os problemas que podem causar.

\section{IMAGEM or: PÍLULA DE EMERGÊNCIA}

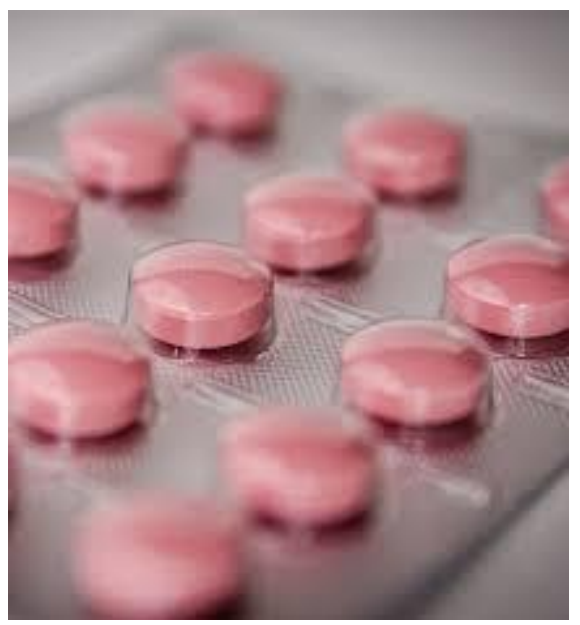

FONTE:https://gi.globo.com/google/amp/pr/parana/especial-publicitario/clinipam/guia-dasaude/noticia/2019/II/28/seis-perguntas-e-respostas-sobre-a-pilula-do-dia-seguinte.ghtml

Mulheres em todo o mundo querem evitar a gravidez e procuram a forma ideal de implementar o planejamento familiar. Os métodos anticoncepcionais são diversos 
e devem ser adaptados às necessidades específicas de cada mulher. As prescrições médicas são essenciais para determinar o tipo mais eficaz. Esses métodos incluem retirada, abstinência e uso de pílulas, por meio de preservativos, dispositivos intrauterinos (DIU), ligadura da trompa de Falópio feminina e vasectomia masculina, Até mesmo os anticoncepcionais, os anéis vaginais e as próprias pílulas anticoncepcionais são usados com frequência.

A pílula é composta de hormônios que estão presentes nos anticoncepcionais convencionais, mas em dose bem maior, e sua função é prevenir a liberação de óvulos e retardar a fertilização. Se a ovulação ocorreu, sua função é descascar o endométrio, o que causa sangramento e impede a implantação do embrião.

Seu efeito não é tão bom quanto o dos anticoncepcionais comumente usados na vida diária e varia com o tempo de uso. A equação é a seguinte: quanto maior o atraso na ingestão, maior a chance de gravidez.

\section{ENOVID-Io: PRIMEIRO PASSO}

Surgindo nos Estados Unidos da América o primeiro contraceptivo da história. No dia I8 de agosto de I96o foi lançado o contraceptivo oral Enovid-ıo e foi anunciada no mercado Alemão.

IMAGEM 02: ENOVID-Io

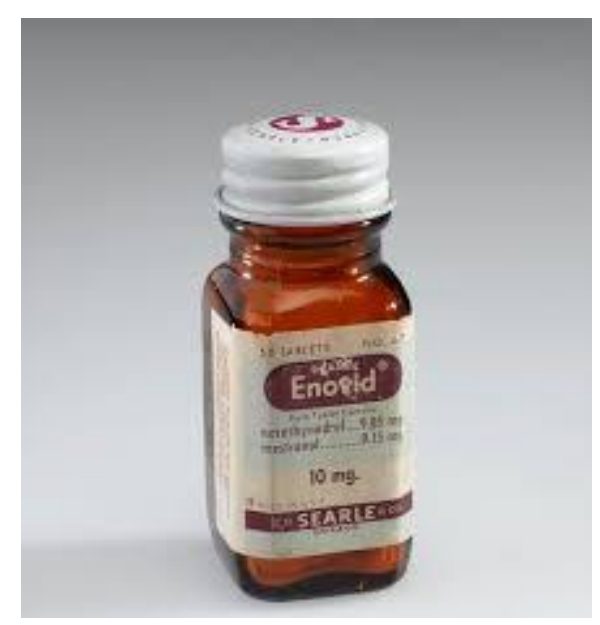

FONTE: https://time.com/392997//enovid-the-pill/?amp=true 
A feminista Margaret Sanger e a milionária Katherine McCormick haviam se unido para inventar uma pílula contra a gravidez que fosse fácil de usar, eficiente e barata. Mesmo com o combinado do projeto, o cientista Gregory Pincus que havia aceitado o projeto teve que trabalhar nas surdinas, pois os contraceptivos eram estritamente proibidos nos Estados Unidos da América até 1965. Ele disse que este é um estudo para aliviar os sintomas menstruais e renunciou cinco anos após o início do estudo. Em i8 de agosto de 196o, lançou um novo produto no mercado americano: o Enovid-ı.

Naquela época, o sexo era considerado apenas uma forma de reprodução. A pílula anticoncepcional significa uma mudança no sexo, pois o casal pode começar a fazer sexo apenas por prazer. Desde 1965, a demanda alemã aumentou muito.

Com a ascensão de Woodstock e dos hippies, o movimento estudantil e o avanço do feminismo, a pílula anticoncepcional atingiu seu auge. No entanto, em um curto período de tempo, efeitos colaterais como desconforto e ganho de peso começaram a se tornar aparentes.

O laboratório continuou a pesquisar e fabricar micropapilas e micropapilas (doses hormonais menores), pílulas matinais, adesivos e implantes hormonais.

A primeira versão do medicamento só chegou no Brasil quase 40 anos depois de seu lançamento, em julho de 1999.

\section{I REAÇÕES NO ORGANISMO}

Os anticoncepcionais de emergência (CE) consistem em compostos hormonais concentrados e são usados para fins de curto prazo. Eles podem interromper a ovulação e a migração dos espermatozoides alguns dias após a relação sexual e têm certas indicações para situações especiais. Indicações incluem relação sexual sem uso de métodos anticoncepcionais, falha de métodos convencionais, abuso de métodos anticoncepcionais e violência sexual (Figueiredo; Bastos, 2008).

Com hormônios adicionais, ele retarda a ovulação, impede a fertilização e não permite que o óvulo se estabeleça no útero se já tiver encontrado o esperma. Portanto, as pílulas anticoncepcionais podem prevenir a gravidez indesejada. $\mathrm{O}$ kit de primeiros 
socorros consiste em duas pílulas contendo altas doses de hormônios sintéticos, como progesterona e estrogênio. No entanto, a primeira pílula só tem efeito 72 horas após a mulher fazer sexo. A segunda dose deve chegar após 12 horas. Quanto maior o atraso, pior o efeito.

As opções que existem atualmente no mercado são de levonogestrel, tanto em dose única $(1,5 \mathrm{mg})$ ou de duas tomadas $(0,75 \mathrm{mg})$, que é um tipo de progesterona sintética” (Murayama, Bárbara)

As pílulas anticoncepcionais podem ter efeitos diferentes. "Pode inibir ou apenas atrasar a ovulação. Isso ocorre porque esse hormônio retarda o movimento da trompa de Falópio, que é onde o óvulo encontra o esperma do ovário para o útero - o esperma encontra o óvulo do útero através da trompa de Falópio. Então isso também torna o espermatozoide difícil de penetrar no muco cervical, porque a progesterona altera a consistência do muco, tornando o ambiente desfavorável para os espermatozoides.”

O muco cervical é um fator importante para a fertilização do óvulo (ou não) e reflete diretamente as alterações hormonais. De modo que, para quem usa anticoncepcional, a consistência é diferente da que não usa. "Uma das funções normais do muco é facilitar a passagem dos espermatozóides pelo óvulo. Nas pessoas que usam métodos hormonais, essa combinação torna o muco mais espesso e dificulta a passagem dos espermatozoides"(Murayama, Bárbara).

\subsection{VANTAGENS DA PÍLULA DO DIA SEGUINTE}

- É o único método contraceptivo que pode ser usado após as relações sexuais;

- Caso não funcione, não causa nenhuma sequela ao feto;

- Previne gravidez não planejada;

- Não tem idade mínima para poder tomar;

- Já se pode tomar assim que se inicia a vida sexual;

- A idade máxima vai até o fim da vida fértil da mulher.

\subsection{DESVANTAGENS DO USO}

- Uso em excesso desregulado o ciclo menstrual e o período fértil da mulher; 
- Grande chance de ter relação sexual desprotegida em dias férteis, facilitando a gravidez;

- Aumenta o risco de gravidez ectópica se usada mais de uma vez em um mês;

- Caso faça um grande uso do contraceptivo emergencial pode prejudicar o funcionamento do reprodutor feminino e dificulta futuras gravidez desejadas;

- Taxa de falha de 5\%;

- Tem vários efeitos colaterais.

\subsection{EFEITOS COLATERAIS}

- Náuseas;

- Diarréia;

- Dor de cabeça;

- Dores no corpo;

- Vômito;

- Tontura;

- Cansaço;

- Sangramentos fora do período menstrual;

- Sensibilidade nos seios.

- Menstruação irregular

3 IMPORTÂNCIA DA ATENÇÃO FARMACÊUTICA: PÍLULA DO DIA SEGUINTE

A assistência farmacêutica é um conjunto de ações realizadas pelo farmacêutico com o objetivo de prevenir, descobrir e solucionar problemas relacionados aos medicamentos e promover seu uso racionalır. Por meio dessa atenção, o farmacêutico tem a responsabilidade compartilhada pela qualidade de vida do paciente.

Os farmacêuticos podem atuar em diversas áreas, principalmente dispensando medicamentos em farmácias, com o objetivo de prestar serviços que ajudem a melhorar a eficácia dos tratamentos medicamentosos, aliando o conhecimento técnico dos medicamentos às condições clínicas dos pacientes.

O CE está facilmente disponível nas farmácias, por isso é muito importante que o farmacêutico possa esclarecer possíveis dúvidas. O farmacêutico possui amplo 
conhecimento sobre farmacodinâmica e farmacocinética e tem papel fundamental na promoção do uso racional de medicamentos, evitando a autoadministração e possíveis reações adversas.

Algumas normas auxiliam o farmacêutico a assumir seu verdadeiro papel de promotor de saúde e garantem o direito das pessoas à assistência médica e farmacêutica. São eles: RDC Anvisa nº 44/2009, que disponibiliza boas práticas farmacêuticas para o controle higiênico das funções, distribuição e comercialização dos produtos, bem como a prestação de serviços farmacêuticos em farmácias e farmácias.

TABELA r: INFORMAÇÕES SOBRE O CONTRACEPTIVO EMERGÊNCIAL

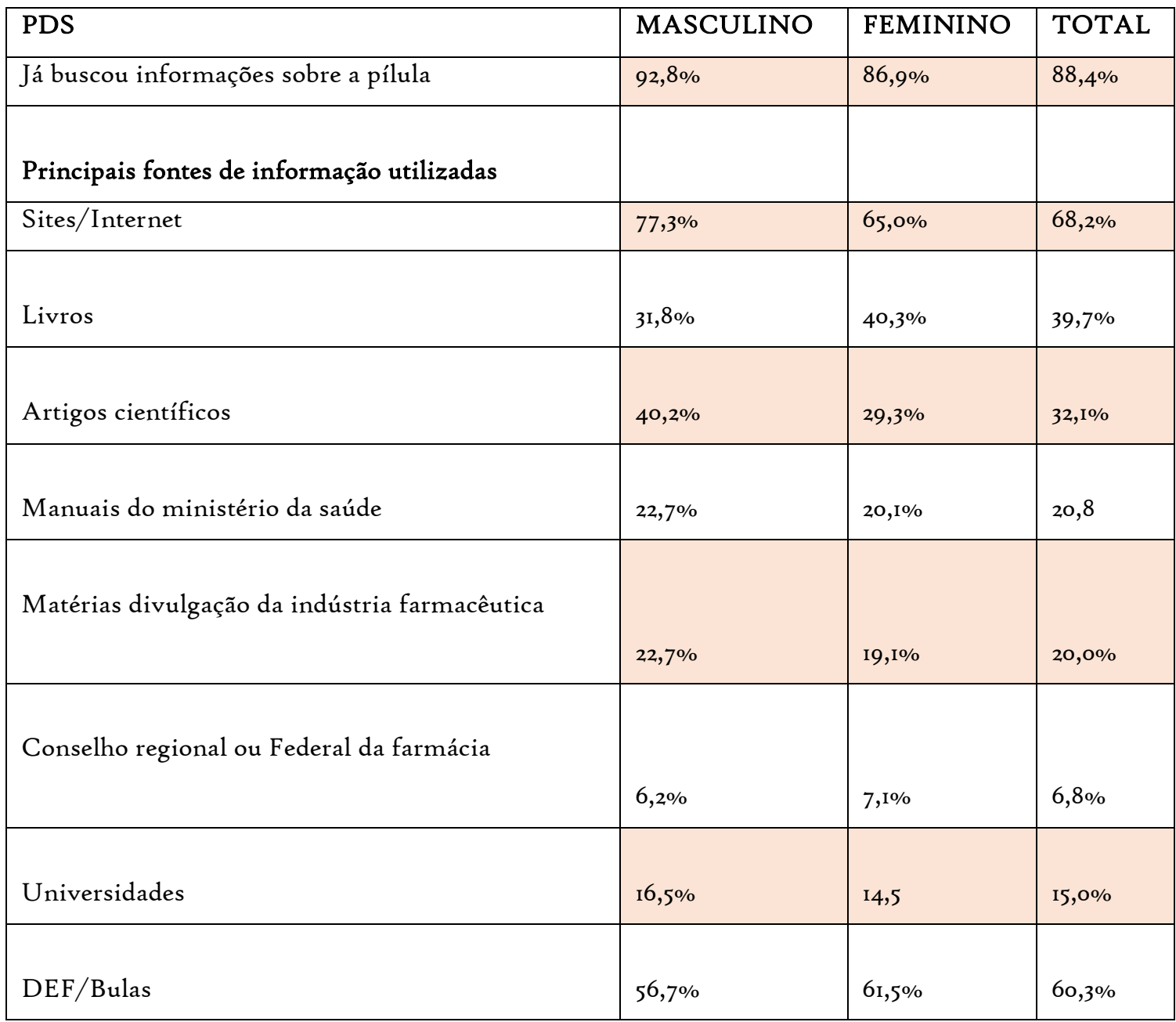

FONTE: Pesquisa uma investigação socioantropológica em farmácias: posição de farmacêuticos e balconistas sobre a contracepção de emergência, Lesc-UFRJ, 2014. 


\section{CONCLUSÃO}

Embora o mecanismo de ação ainda seja bastante complexo, é sabido que ele varia muito, dependendo do momento do ciclo menstrual em que o anticoncepcional é administrado. Se a pílula for tomada logo após a menstruação, ela mudará o desenvolvimento dos folículos ovulatórios, de forma que a ovulação não ocorrerá ou retardará a ovulação e, em quase $85 \%$ dos casos, os espermatozoides não terão a oportunidade de tocar o óvulo. Isso evita a fertilização.

A pílula anticoncepcional também pode atrasar o transporte dos espermatozoides no tubo, tornando-os mais lentos. Outro mecanismo de ação é engrossar o muco do colo do útero e dificultar a migração dos espermatozoides para o útero. Essa terapia também altera a capacitação dos espermatozoides, que é um processo importante pelo qual o espermatozoide pode fertilizar um óvulo. Uma vez que a capacitação é alterada, ele não será fertilizado.

A pílula anticoncepcional não é recomendada como método anticoncepcional. Seu uso só deve ser realizado em situações de emergência. Para evitar gravidez indesejada e doenças sexualmente transmissíveis, você pode escolher diferentes métodos de acordo com seu corpo e necessidades, e sempre sob a supervisão de um ginecologista.

\section{REFERÊNCIAS}

POR GUIA DA FARMÁCIA, 23 DE SETEMBRO DE 2019, COMO SABER SE A PÍLULA DO DIA SEGUINTE FUNCIONOU? Disponível em: https://guiadafarmacia.com.br/como-saber-se-a-pilula-do-dia-seguinte-funcionou/ Acessado em or/o6/2021

POR CALENDÁRIO HISTÓRICO, I8 DE AGOSTO DE 2020, 1960: PRIMEIRA PÍLULA ANTICONCECIONAL CHEGA AO MERCADO. Disponível em: https://amp.dw.com/pt-br/196o-primeira-p\%C3\%ADlula-anticoncepcional-chegaao-mercado/a-6ir248 Acessado em oI/o6/2021

SABRINA PEREIRA PAIVA, ELAINE REIS BRANDÃO, 25 DE FEVEREIRO DE 2OII, CONTRACEPÇÃO DE EMERGÊNCIA NO CONTEXTO DAS FARMÁCIAS: REVISÃO CRÍTICA DE LITERATURA. Disponível em: https: //www.google.com.br/url? sa =t\&source=web\&rct=j\&url=https: $/ /$ www.scielo.b 
r/pdf/physis/v22ni/v22niao2.pdf\&ved=2ahUKEwiGgfnrpfXwAhWqK7kGHWviA i8QFjABegQIDRAC\&usg=AOvVawoVihPc4htd2X4 $\mathrm{X}_{\text {reacooh6 Acessado em }}$ $02 / 06 / 2021$

DR. PEDRO PINHEIRO, 3 DE JUNHO DE 202I, PÍLULA DO DIA SEGUINTE COMO TOMAR E EFICÁCIA. Disponível em: https://www.mdsaude.com/ginecologia/anticoncepcionais/pilula-dia-seguinte/ Acessado em 05/06/2021

POR GINECO, I6 DE MARÇO DE 2021, PÍlULA DO DIA SEGUINTE. Disponível em: https://www.gineco.com.br/saude-feminina/materias-2/pilula-dodia-seguinte Acessado em 03/06/2021 Acessado em 06/o6/2021

POR GABRIELA KIMURA, 26 DE ABRIL DE 2016, COMO A PÍLULA DO DIA SEGUINTE AGE NO CORPO? Disponível em: https://www.google.com.br/amp/s/claudia.abril.com.br/saude/como-a-pilula-dodia-seguinte-age-no-corpo/amp/?espv=I Acessado em 04/o6/202I

POR REDAÇÃO MUNDO ESTRANHO, I8 DE ABRIL DE 20II, COMO FUNCIONA A PÍLULA DO DIA SEGUINTE? Disponível em: https://www.google.com.br/amp/s/super.abril.com.br/mundo-estranho/comofunciona-a-pilula-do-dia-seguinte/amp/?espv=I Acessado em 04/o6/202I

POR REDAÇÃO MINHA VIDA, oI/II/2020, PÍLULA DO DIA SEGUINTE: VALOR, NOMES E COMO TOMAR. Disponível em: https://www.minhavida.com.br/saude/tudo-sobre/31795-pilula-do-dia-seguinte Acessado em 05/06/2021

AMANDA VIEIRA LEAL, CAMILLA RODRIGUES E RODRIGUES, MAGNA FARDIM DALCIN, I2 DE JUNHO DE 2019, ATENÇÃO FARMACÊUTICA NO USO DE CONTRACEPTIVOS DE EMERGÊNCIA: UMA BREVE REVISÃO. Disponível em: https://www.google.com.br. Acessado em o6/o6/202I

GIULIA GRANCHI, in DE DEZEMBRO DE 2020, PÍLULA DO DIA SEGUINTE: QUAIS SÃO OS RISCOS? ATÉ QUANDO FAZ EFEITO? Disponível em: https://www.uol.com.br/vivabem/faq/pilula-do-dia-seguinte-quais-sao-os-riscosposso-tomar-ate-quando.htm Acessado em 06/06/2021. 